\title{
Las creencias de partida del profesorado en formación en la Enseñanza del Español como Lengua Extranjera (ELE) sobre la gramática
}

The initial beliefs about grammar from teachers in training Teaching Spanish as a second Language (SFL)

\section{Volumen 17, Número 3}

Setiembre-Diciembre

pp. 1-19

Este número se publica el $1^{\circ}$ de setiembre de 2017

DOI: https://doi.org/10.15517/aie.v17i3.30154

Félix Villalba Martínez

Diana Hincapié Moreno

Guillermo Molina Morales

Revista indizada en REDALYC, $\underline{\text { SCIELO }}$

Revista distribuida en las bases de datos:

LATINDEX, DOAJ, REDIB, IRESIE, CLASE, DIALNET, SHERPA/ROMEO, QUALIS-CAPES, MIAR

Revista registrada en los directorios:

ULRICH'S $, \underline{R E D I E}, \underline{\text { RINACE}}, \underline{\text { OEI }}, \underline{\text { MAESTROTECA }}, \underline{\text { PREAL, }}$ 


\title{
Las creencias de partida del profesorado en formación en la Enseñanza del Español como Lengua Extranjera (ELE) sobre la gramática
}

The initial beliefs about grammar from teachers in training Teaching Spanish as a second Language (SFL)

\author{
Félix Villalba Martínez ${ }^{1}$ \\ Diana Hincapié Moreno² \\ Guillermo Molina Morales ${ }^{3}$
}

\begin{abstract}
Resumen. En las últimas décadas ha cobrado protagonismo el estudio de las creencias del profesor y de la profesora en el ámbito de las lenguas extranjeras por su influencia en la práctica docente. En el presente artículo, se analizaron las creencias de 30 docentes colombianos en formación (en el Instituto Caro y Cuervo de Bogotá, donde se realiza el estudio), acerca de la enseñanza de gramática del Español como Lengua Extranjera (ELE). El objetivo fue avanzar en la comprensión de estas cogniciones, y así elaborar programas de formación más adecuados para el contexto estudiado. Se optó por un abordaje mixto que permite la triangulación entre los datos obtenidos por medio de instrumentos cuantitativos (cuestionario) y cualitativos (entrevistas grupales $e$ individuales). Las categorías de análisis fueron las siguientes: concepción de la gramática, experiencia como aprendientes de idiomas, enseñanza de la gramática, aprendizaje de la gramática y práctica docente. Los resultados mostraron una aceptación del enfoque comunicativo, aunque con pervivencia de una visión tradicional en la concepción de la gramática. En varias de las categorías, los porcentajes indicaron la preferencia de las personas docentes hacia actividades que permitieran una interacción real con la lengua, a pesar de que no siempre se precisa la manera de llevarlo a cabo. En el grupo estudiado, se percibió el alejamiento de la visión tradicional sobre el proceso de enseñanza/aprendizaje de la gramática, lo cual supone una relativa novedad respecto a estudios anteriores. Los resultados invitan a elaborar cursos de formación que incidan específicamente en el tratamiento didáctico de la enseñanza de la gramática, por ser el aspecto más desconocido en los futuros profesionales de la enseñanza.
\end{abstract}

Palabras clave: creencias, enseñanza de la gramática, formación de docentes, enseñanza de una segunda lengua

\begin{abstract}
In recent decades, studies in foreign language teaching have shifted their focus to the teacher's beliefs, due to their influence in classroom practices. This study analyzed the beliefs about grammar of 30 Colombian teachers, training as teachers of grammar of Spanish as a foreign language (SFL). The study was aimed at understanding a teacher's cognition in order to elaborate more effective training programs. This research surveyed data by quantitative (questionnaire) and qualitative methods (individual and group interviews). The categories of analysis were: conception of grammar, experience as language learners, teaching grammar, learning grammar, and teaching practicum. The results show an acceptance of the communicative approach, although there is persistence of a traditional view in the conception of grammar. In several of the categories, results indicate a preference towards activities that allow real interaction with the language, though a way to carry it out is not clearly defined. A departure from the traditional view of the teaching / learning grammar is perceived, setting this study apart from other results in previous studies. These results suggest the need to develop training courses that target specifically the didactic teaching of grammar, being the most unfamiliar aspect for prospective teachers.
\end{abstract}

Key words: beliefs, grammar teaching, teacher training, second language instruction.

\footnotetext{
${ }^{1}$ Director de Investigación en ELE/2 y asesor de la Dirección General del Instituto Caro y Cuervo, Bogotá, Colombia. Doctor en didáctica de la Lengua y la Literatura por la Universidad Complutense de Madrid, España.Dirección Electrónica: felix.villalba@caroycuervo.gov.co
}

${ }^{2}$ Docente e investigadora del Instituto Caro y Cuervo, Bogotá, Colombia. Máster en Neuropsicología y Educación por la Universidad Internacional de la Rioja, España. Dirección Electrónica: Diana.hincapie@caroycuervo.gov.co

${ }^{3}$ Docente e investigador del Instituto Caro y Cuervo, Bogotá, Colombia. Doctor por la Universidad de Zaragoza, España.Dirección Electrónica: Guillermo.Molina@caroycuervo.gov.co

Artículo recibido: 19 de octubre, 2016

Enviado a corrección: 22 de mayo, 2017

Aprobado: 24 de iulio, 2017 


\section{Introducción}

El interés por el estudio de las creencias en el ámbito educativo se enmarca dentro del paradigma cognitivista que desplazó el foco de estudio desde las prácticas observables de docentes hacia las ideas que podían sustentarlas (Gabillon, 2013). De este modo, la investigación en creencias se consideró importante para conocer mejor las percepciones, juicios y prácticas de los docentes e intervenir sobre ellas para modificarlas (De Witt et al., 1998).

Las creencias, por lo tanto, se incluyen dentro de un concepto más amplio conocido como "cogniciones" o "representaciones mentales" de la persona docente ("teacher cognition"); término que hace referencia al conjunto de creencias, conocimientos, principios, teorías, actitudes y reflexiones del propio profesorado a lo largo del proceso de enseñanza (Borg, 2003). Las creencias pueden relacionarse con diferentes contenidos que intervienen en la práctica docente: sobre la lengua en general, sobre el aprendizaje de la L2, sobre la enseñanza de la gramática, sobre el estudiantado, etc.

Entre las características que singularizan las creencias del profesorado, la revisión bibliográfica destaca las siguientes: son dinámicas, pueden modificarse por la reflexión, forman sistemas complejos y dialécticos, están íntimamente relacionadas con factores afectivos y emocionales, son producto de un determinado contexto sociocultural, pueden presentar inconsistencias y conflictos, etc. (Barcelos y Kalaja, 2011; Borg, 2006; Wu, 2006).

Con estas características podemos llegar a una definición integradora y operativa del concepto de "creencias", como la que propone Ramos (2007):

Las creencias son ideas relativamente estables que tiene un individuo sobre un tema determinado, forjadas a través de su experiencia personal bajo la influencia de un proceso de construcción social, agrupadas en redes o sistemas, de cuya veracidad está convencido y que actúan como un filtro a través del cual percibe e interpreta el mundo que lo rodea, tomando sus decisiones de acuerdo con ello. (p. 21)

En la actualidad, el estudio de las creencias destaca sus componentes socioculturales y contextuales. Desde esta perspectiva, se entienden como construcciones sociales que presentan una notable variación con respecto a los estilos pedagógicos con los que se entra en contacto, al significado de los contenidos de aprendizaje y a los modelos de interacción social e institucional, etc. (Eisenstein-Ebsworth y Schweers, 1997). 
Es precisamente esta dimensión la que orienta, en parte, el presente estudio referido al contexto colombiano y, más específicamente, al bogotano. La investigación pretende identificar las creencias que poseen 30 docentes en formación sobre la gramática y su proceso de enseñanza-aprendizaje. Este trabajo se enmarca, por lo tanto, en el área de los estudios que intentan identificar las creencias docentes para así afinar los programas de formación continua (Clarke y Hollingsworth, 2002).

\section{Referente teórico}

Las investigaciones sobre creencias en el área de enseñanza-aprendizaje de lenguas partieron desde el interés por conocer la cognición del estudiantado hasta llegar a los estudios sobre las creencias de las personas docentes (Borg, 2006). Del mismo modo, el foco de estudio pasó de querer identificar lo que estudiantes y docentes creían sobre la lengua, en la década de los 80, a intentar comprender cómo se conforman las creencias.

Barcelos y Kalaja (2011) presentan una interesante síntesis sobre los estudios acerca de las creencias de las profesoras y los profesores. Para estas investigadoras, los diferentes trabajos han seguido tres enfoques claramente diferenciados: el normativo, el metacognitivo y el contextual. La primera orientación posee un cierto carácter determinista, pues se considera que las creencias influyen de manera directa en las futuras conductas de quienes enseñan. El enfoque metacognitivo concibe las creencias como fruto de este tipo de conocimientos, aunque entendidas como características individuales, subjetivas y $\sin$ relación con el conocimiento formal. Por último, el enfoque contextual entiende las creencias estrechamente relacionadas con el contexto en el que se producen y, en ese sentido, intentan tener en cuenta la perspectiva docente (emic).

Por otro lado, los estudios sobre creencias, en el área de enseñanza de lenguas, también se pueden clasificar según el objeto de las investigaciones. En este sentido, Gabillon (2013) distingue cinco líneas de trabajo: relaciones entre creencias del profesorado y práctica docente, discrepancias entre creencias del profesorado y creencias de estudiantes, naturaleza de las creencias del profesorado, creencias sobre innovación educativa y estudios que investigan las creencias del profesorado como fuente para su concienciación y crecimiento profesional.

La presente investigación se enmarca en la última de estas líneas. De esta manera, se considera que el estudio de las creencias facilita la autorreflexión y autodesarrollo de las personas docentes. Los objetivos generales de este tipo de investigaciones recaen en 
ayudar al personal docente a tener conciencia sobre las relaciones que existen entre sus creencias y su actuación docente (Farrell y Lim, 2005).

\subsection{Creencias sobre gramática}

Los estudios de creencias respecto a la enseñanza de gramática han seguido tres líneas principales de investigación: el conocimiento declarativo de gramática, las creencias expresadas sobre enseñanza de gramática y las ideas reflejadas en sus prácticas de enseñanza (Borg, 2003).

La primera de estas líneas se refiere tanto a lo que las personas docentes creen que saben sobre gramática como a lo que realmente conocen. También, dentro de estos estudios se han querido identificar los conocimientos de partida adquiridos durante el periodo de formación y su evolución con la práctica docente.

Sobre el conocimiento de la gramática por parte del profesorado y futuros docentes, los estudios revelan que, por lo general, el nivel de dicho conocimiento suele ser muy bajo (Williamson y Hardman, 1995). Al mismo tiempo se da un mayor nivel de conocimiento gramatical entre docentes no nativos que entre nativos, los niveles más bajos corresponden a estudiantes sin graduar en periodo de formación (Andrews, 1999).

En cuanto a la influencia de la experiencia previa, y pese a lo que pudiera esperarse, no siempre, el dominio de aspectos gramaticales aumenta con el tiempo. Andrews (1999) constata que el tiempo de práctica docente no afecta al cambio. Berry (2014) se centra en el uso de metalenguaje (entendido como el lenguaje que se utiliza para hablar de gramática) y, en un estudio longitudinal de más de diez años, encuentra una desigual evolución en función de los intereses y el desarrollo profesional personal. Borg afirma que "aquellos maestros que no buscan el conocimiento no lo consiguen" (2005, p. 16).

La (in)seguridad de las personas docentes sobre su propio conocimiento gramatical influye de manera decisiva en la toma de decisiones y en la práctica docente. Borg (1999) ilustra este comportamiento cuando comenta que es frecuente encontrar docentes que no enseñan mucha gramática por temor a cometer equivocaciones o a que el estudiantado les sorprenda con preguntas comprometedoras.

Las investigaciones realizadas en el marco de la segunda orientación antes mencionada, referida a las creencias expresadas sobre enseñanza de gramática, ponen de manifiesto una gran discrepancia entre los enfoques didácticos recomendados por los especialistas y las creencias actuales de quienes enseñan. Chandler, Robinson y Noyes 
(1988) llegan a afirmar que existe una "ignorancia confiada" que permite la reproducción de prácticas docentes "pasadas de moda", provenientes de la experiencia previa como estudiante. De forma análoga, Burgess y Etherington (2002) concluyen que la mayoría de docentes todavía cree en la eficacia de la enseñanza tradicional de la gramática. Por su parte, Eisenstein-Ebsworth y Schweers (1997) puntualizan que este tipo de creencias es más frecuente en algunos contextos culturales pedagógicos, como el del profesorado de inglés de Puerto Rico respecto a sus homólogos de Nueva York.

La revisión bibliográfica también pone en evidencia el desfase entre las creencias de docentes y las realidades de sus estudiantes. Por ejemplo, Berry (1997) encontró que el profesorado tiende a sobrevalorar los conocimientos de sus discentes sobre metalenguaje. Por su parte, Schulz (2001) llegó a la conclusión de que, tanto en Estados Unidos como en Colombia, la corrección de errores se considera más importante entre estudiantes que entre las personas que enseñan. En el caso específico de Colombia, en el estudio, llama la atención el hecho de que la mayoría del estudiantado declaraba su gusto por la gramática, algo que no era previsto por sus docentes.

Dentro de esta segunda línea, también se ha intentado investigar acerca de la propia naturaleza de las creencias y, más concretamente, del modo en el que la experiencia previa del profesorado influye en su formación. Dentro de esta experiencia, Borg (1999) identifica tres ámbitos claramente diferenciados: la escolarización previa del personal docente (en el papel de aprendices), la participación del profesorado en programas específicos de formación y la trayectoria docente.

Finalmente, la última de las líneas de investigación que explora la relación entre las creencias sobre gramática y la práctica en el aula del profesorado ha sido ampliamente estudiada (Borg, 2001). Santiago (2010) distingue entre aspectos contextuales generales (programa de la materia, requisitos de la institución, etc.) y aspectos personales del profesorado. Respecto a los aspectos contextuales, destaca el estudio de Johnston y Goettsch (2000), en el que se constata que el tratamiento de la gramática en clase suele depender del nivel del curso con el que se esté trabajando.

En cuanto a los componentes personales, Pahissa y Tragant (2009) estudian a tres profesoras no nativas con experiencia y encuentran que su tratamiento de la gramática está influido por su experiencia en el aprendizaje del idioma y por la falta de confianza en el dominio de la L2. Esta falta de confianza se traduce en un mayor apoyo en la gramática dentro del aula (Berry, 2014) y, al mismo tiempo, en una disminución del metalenguaje 
empleado. Frente a esto, Nazari y Allahyar (2012) reportan un comportamiento interesante de una profesora que recurre a explicaciones gramaticales ricas en metalenguaje para reforzar su autoridad y conseguir que el estudiantado valore la calidad de sus clases.

Por último, los conocimientos acerca de la gramática y su enseñanza se manifiestan, entre otras, en las siguientes decisiones que toman a diario las personas docentes: cómo organizar la estructura formal de la lección, qué contenidos destacar, cómo seleccionar las actividades de aprendizaje, cómo corregir, etc.

Es importante aclarar que en estos estudios, así como en el que nosotros realizamos, los investigadores no toman como punto de partida una determinada perspectiva gramatical (generativa, funcionalista, cognitiva, etc.). En todo caso, el objetivo sería acercarse a la visión del estudiantado, sin sesgos iniciales. En este tipo de análisis, enfocados en la práctica educativa, interesa más la distinción entre los enfoques para la enseñanza de la gramática: si el profesorado propende a un aprendizaje implícito o explícito, memorístico o comunicativo, por ejemplo. Hacia esto se dirige la mayoría de las cuestiones de nuestra investigación.

\section{Metodología}

\subsection{Sujetos}

En el presente estudio participaron un total de 30 docentes bogotanos en formación, con edades comprendidas entre los 23 y 50 años de edad, estudiantes del "Diplomado en pedagogía y didáctica para la enseñanza de español como lengua extranjera" del Instituto Caro y Cuervo. Dichos sujetos componen la totalidad de la primera cohorte del citado diplomado durante el año 2016. La mayoría de ellos son egresados de programas de licenciatura en enseñanza de lenguas y actualmente ejercen como docentes en educación formal y no formal. Algunos de ellos tienen experiencia como docentes de español como lengua extranjera, y todos han sido aprendientes de algún idioma diferente al materno.

\subsection{Instrumentos}

Kalaja y Barcelos (2003) refieren que la metodología empleada en los diferentes tipos de investigaciones ha evolucionado según de la orientación: de los cuestionarios cerrados sobre creencias propios del enfoque normativo se ha pasado paulatinamente a la triangulación, en la que se incluyen entrevistas e informes individuales, para tener en cuenta los factores contextuales. El presente estudio opta por esta última metodología. 
Así pues, con el propósito de lograr una comprensión más amplia del objeto de estudio, se ha combinado un instrumento de análisis cuantitativo (cuestionario) con dos métodos cualitativos (discusiones grupales y entrevistas individuales). Estos dos últimos instrumentos son concebidos como una forma de ampliar la información registrada en los cuestionarios y situarla en el contexto de la persona docente en formación.

\subsection{Cuestionario}

El cuestionario digital está compuesto por 31 ítems. Como punto de partida se tomaron algunas preguntas de Uysal y Bardakci (2014), aunque posteriormente se elaboraron otras de forma autónoma. Se establecieron las siguientes categorías para clasificar los ítems: a) Cuestiones generales sobre gramática, b) experiencia como aprendiente de idiomas, c) enseñanza de la gramática, d) aprendizaje de la gramática y, e) práctica docente.

Se utilizó una escala Likert con una etiqueta nominal de "muy en desacuerdo" a "muy de acuerdo", y de "poco importante" a "muy importante". Algunas preguntas o enunciados incluidos en el cuestionario son: "La gramática es importante y tiene que ser enseñada", "los ejercicios de drill (completar huecos) son una herramienta muy útil en la enseñanza de la gramática", "los enfoques de la enseñanza de la gramática dependen en gran medida del nivel de competencia de los estudiantes en la L2", "Es muy importante la enseñanza implícita (incidental) de la gramática", "lo que más te ha servido en el aprendizaje de la gramática fue la memorización", "para la enseñanza de la gramática es importante corregir a los estudiantes", etc.

\subsection{Discusiones grupales}

Las discusiones se dieron en un tiempo de 30 minutos, en 5 grupos de 5 personas cada uno (junto a un moderador por grupo) y fueron grabadas. Se debatieron las siguientes afirmaciones:

1. La gramática solo puede ser aprendida mediante las explicaciones directas de la persona docente.

2. Las clases de gramática son aburridas pero necesarias.

3. Lo que nunca haría al enseñar gramática sería...

4. Los problemas más importantes en el aprendizaje de lenguas extranjera son aquellos relacionados con la gramática. 


\subsection{Entrevistas individuales}

Al igual que las discusiones, las entrevistas fueron grabadas. Cada una tuvo una duración de aproximadamente 15 minutos. Las cuestiones planteadas en esta entrevista fueron:

1. ¿Cómo aprendiste la gramática de una lengua extranjera (inglés, francés, etc.)?

2. ¿Qué profesor de LE recuerdas especialmente? ¿Cómo eran sus clases?

3. ¿Qué dificultades crees que van a tener tus estudiantes de ELE/L2 para la gramática?

4. ¿Qué crees que tienen que hacer los estudiantes para aprender gramática?

5. ¿Qué dificultades crees que vas a tener tú al enseñar gramática?

6. ¿Qué necesitarías aprender en este diplomado para mejorar tu futura práctica docente?

7. ¿La exposición a la lengua extranjera es necesaria pues la gramática se adquiere mediante el contacto directo con la L2 o es necesario enseñarla explícitamente?

\section{Resultados y análisis}

En cuanto a la concepción de la gramática, la mayor parte del estudiantado entiende que esta es importante y necesita ser enseñada $\left(M=4.24,86 \%{ }^{4}\right)$, se orienta al estudio de los aspectos formales $(\mathrm{M}=3.62,58 \%)$ y es necesaria para producir en una $\mathrm{L} 2$ ( $\mathrm{M}=3.75,69 \%)$. Por otra parte, en las respuestas, se manifiesta una cierta preocupación por contemplar la actuación de otros tipos de componentes. Así, cerca del $82 \%(M=4.17)$ de las respuestas admiten que la gramática de una L2 se aprende en contacto directo con la comunidad de habla ${ }^{5}$.

Respecto a la reflexión acerca de su propio proceso de aprendizaje de la gramática, la mayor parte de los participantes reconoce que es muy importante el estudio individual $(M=4.17,75.9 \%)$, el entrar en contacto con muestras de lengua reales $(M=4.58)$ y la utilización efectiva de la lengua $(M=4.75)$. En menor medida, conceden cierta relevancia a la memorización $(M=3.58,58 \%)$ y a la realización de ejercicios $(M=3.79,65 \%)$.

Respecto al proceso de enseñanza de la gramática, existe una clara coincidencia en cuestionar el uso de la LM en clase para las explicaciones gramaticales, así como el uso del

\footnotetext{
${ }^{4}$ En todo el texto, los porcentajes se refieren a los docentes que se muestran "de acuerdo" o "muy de acuerdo" con la afirmación.

5 Tanto para el reconocimiento de la importancia de la enseñanza de la gramática como para la idea de que la gramática se aprende en contacto con la L2, los resultados obtenidos son bastante fiables, con un intervalo de confianza de alrededor de 0,26 en ambos casos (0,26 en el primero y 0,27 en el segundo).
} 
metalenguaje. Solo un $47 \%$ apoya esta práctica de la LM y apenas un $27 \%$ respalda el uso del metalenguaje.

En las reflexiones acerca de los procesos de enseñanza no existe claridad acerca de la conveniencia del uso o no de los ejercicios de drill, solo un $37 \%$ los respalda frente a un $31 \%$ que los cuestiona. Frente a estos datos existe un marcado respaldo al uso de actividades comunicativas ( $\mathrm{M}=4.0,69 \%)$.

En cuanto a si la enseñanza de la gramática ha de ser mediante procedimientos explícitos o implícitos, la mayor parte del estudiantado se decanta por esta última opción $(M=4.17,82 \%)$ frente a la enseñanza explícita $(M=3.37,37 \%)$. En relación con estos datos, reconocen la importancia de prestar atención a la forma de la lengua $(M=4.31,89 \%)$ y también a su uso $(\mathrm{M}=4.79,96 \%)$.

Por último, los participantes no respaldan abiertamente las ideas acerca de que los enfoques metodológicos varíen respecto al nivel del estudiantado ( $M=3.65,51 \%)$ o que en el ámbito académico haya que enseñar gramática $(\mathrm{M}=3.51,58 \%)$.

Respecto al papel del profesorado en la práctica docente, se entiende que su papel es fundamentalmente el de corregir $(\mathrm{M}=4.24,82 \%)$ y el de proporcionar retroalimentación al estudiantado ( $M=4.86,100 \%)$. Sin embargo, no existe tal claridad respecto a cómo hacerlo: sólo el $31 \%$ apoya la idea de que se haga mediante explicaciones de la persona docente.

Para terminar, y en cuanto al papel del profesorado en el aula, apenas un 3.17 de promedio se inclina por una secuencia didáctica clásica en la que a las explicaciones gramaticales les siguen prácticas controladas, mientras que el $M=3.82$ reconoce la importancia de la reflexión gramatical de los propios estudiantes (65\%). Existe una clara coincidencia en reconocer que la persona docente tiene que dominar los contenidos gramaticales $(M=4.89)$. Al mismo tiempo, las personas participantes reconocen que el estudiante ha de desempeñar un papel activo en la construcción del conocimiento $(M=4.68)$ y que es importante que ponga en práctica lo aprendido $(M=4.96)$. Estos datos se suman al hecho de que se cuestiona el papel central del libro de texto $(M=3.06)$, y se reconoce abiertamente la importancia del clima afectivo ( $M=4.10,76 \%)$.

Los resultados obtenidos, tanto los de naturaleza cuantitativa (derivados de la encuesta) como los cualitativos (entrevistas grupales e individuales), permiten observar que el estudiantado muestra una tendencia hacia el enfoque comunicativo en la enseñanza de gramática, aunque la visión tradicional mantiene un cierto peso en sus concepciones. Así, en las conversaciones grupales aparecen a menudo frases que indican la aceptación del 
enfoque comunicativo: "más que la gramática correcta es la voluntad de comunicación" (grupo 4); "no usaría mucho tiempo ni haría compleja la explicación. Que se sienta que lo más importante de la clase fue la comunicación" (grupo 3). Al mismo tiempo, se reconoce en ocasiones la dificultad de salir de los modelos tradicionales: "a pesar que hoy se habla mucho del enfoque comunicativo aun no somos capaces de despegarnos de la gramática" (grupo 5); "es difícil ser creativo a la hora de enseñar gramática, porque hemos heredado un enfoque formal y ya estamos adaptados" (grupo 2). En las gráficas 1 y 2, podemos percibir la convivencia entre un enfoque más "tradicional" centrado en el aprendizaje, y otro más "novedoso" que incide en los procesos de adquisición:

\section{Gráfica 1}

Distribución porcentual por nivel de acuerdo de los docentes en formación del Instituto Caro y Cuervo de Bogotá, ante la afirmación "La gramática se orienta fundamentalmente hacia el estudio de las formas correctas", 2016. $(n=30)$.

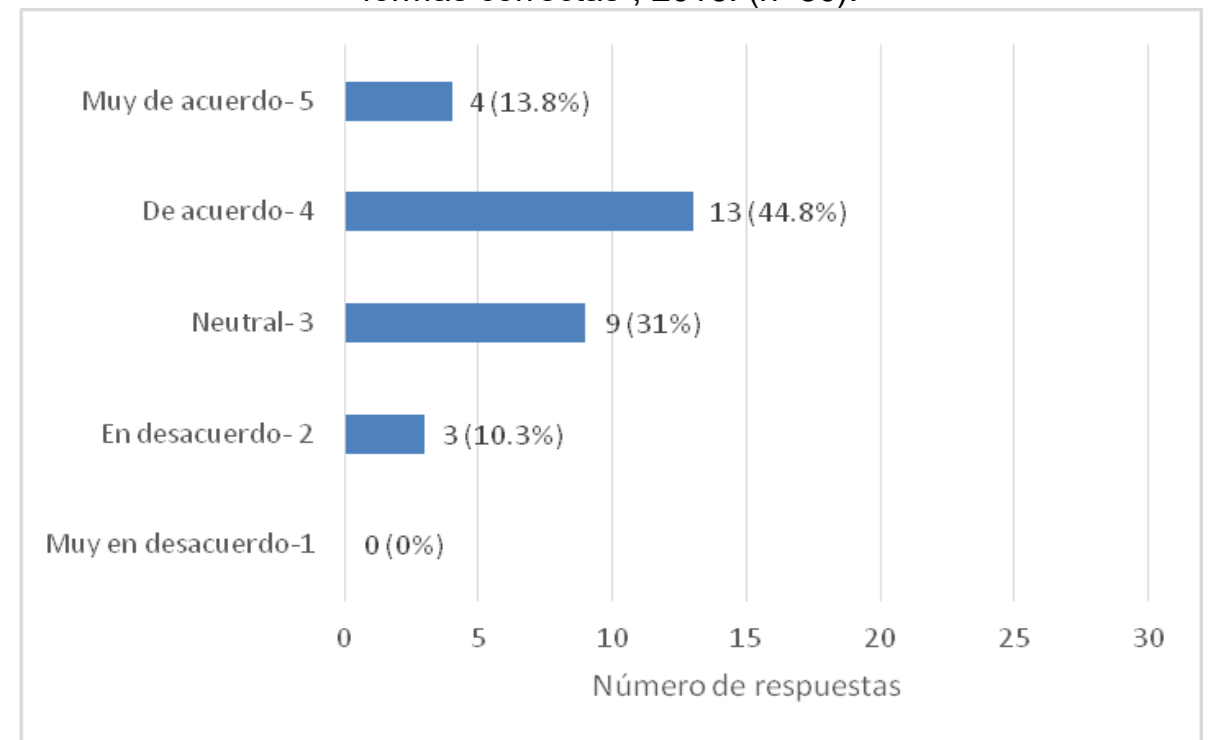

Fuente: Elaboración propia con la información recolectada, 2016. 


\section{Gráfica 2}

Distribución porcentual por nivel de acuerdo de los docentes en formación del Instituto Caro y Cuervo de Bogotá, ante la afirmación "La exposición a la lengua extrajera es muy importante, pues la gramática se adquiere mediante el contacto directo con la LE/2", 2016. $(n=30)$.

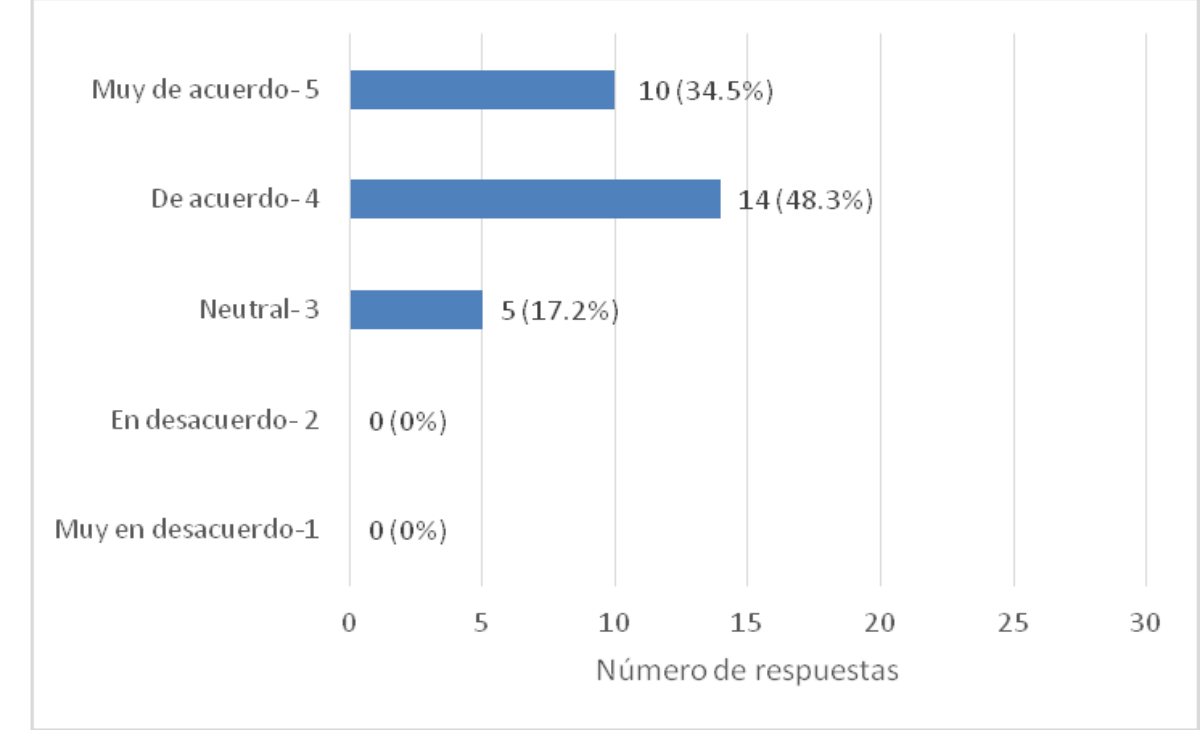

Fuente: Elaboración propia con la información recolectada, 2016.

Esta inclinación matizada se manifiesta en aspectos clave como la elección de una enseñanza implícita o una explícita. El 82,8\% del estudiantado admite que la lengua puede adquirirse en contacto directo con la comunidad de habla y, en consecuencia, respalda de forma mayoritaria la enseñanza implícita ( $M=4.16$ frente a $M=3.37$ de la explícita). Sin embargo, las entrevistas grupales apuntan, en ocasiones, a la necesidad de la enseñanza explícita. Así, en el conjunto de personas entrevistadas, encontramos afirmaciones como "es necesaria la explicación directa del profesor", "es muy difícil que un estudiante deduzca la gramática”, "algunos temas requieren una intervención académica o una explicación”, "hay cosas que explico explícitamente, hay explicaciones que sí se necesitan hacer" o "me ha funcionado más lo explícito" lo que remite a modelos que, en teoría, ya están superados. Esta información se ve representada en las gráficas 3 y 4 : 


\section{Gráfica 3}

Distribución porcentual por nivel de acuerdo de los docentes en formación del Instituto Caro y Cuervo de Bogotá, ante la afirmación "Es muy importante la enseñanza explícita (deliberada) de la gramática", 2016. $(n=30)$.

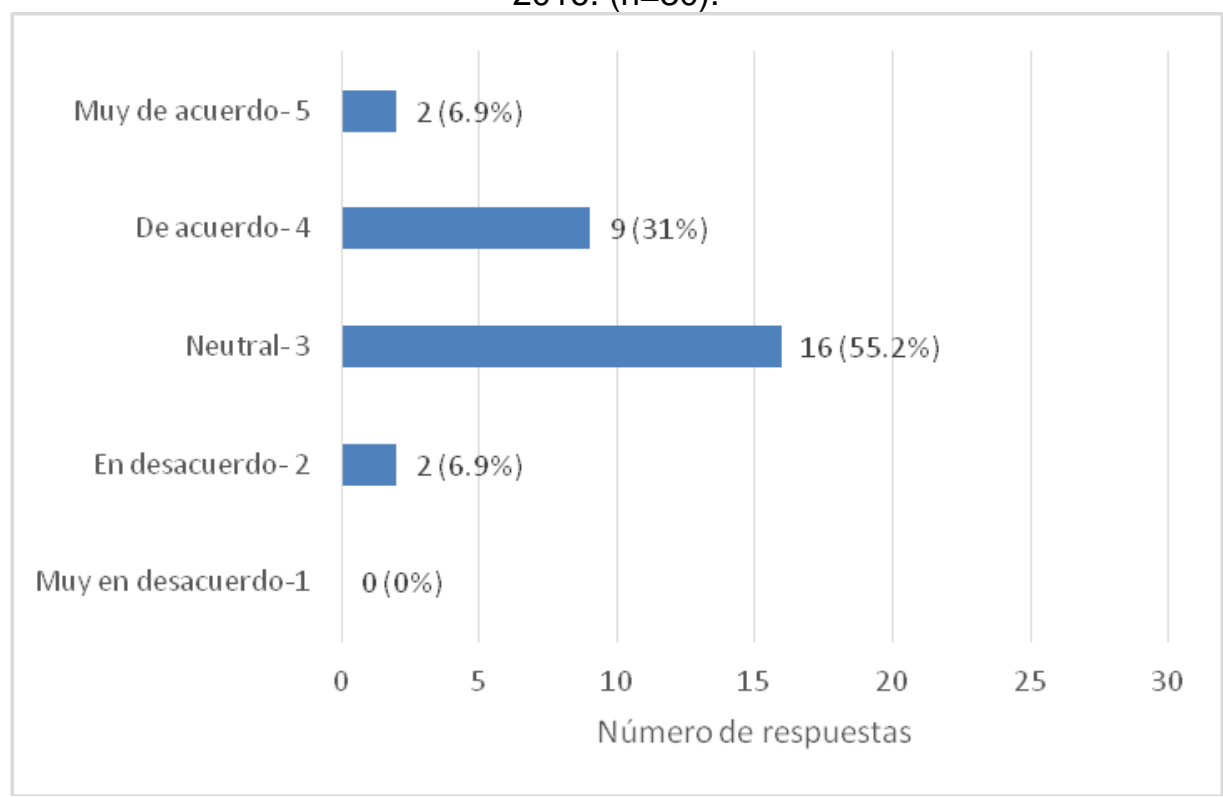

Fuente: Elaboración propia con la información recolectada, 2016.

\section{Gráfica 4}

Distribución porcentual por nivel de acuerdo de los docentes en formación del Instituto Caro y Cuervo de Bogotá, ante la afirmación "Es muy importante la enseñanza implícita (incidental) de la gramática", 2016. $(n=30)$.

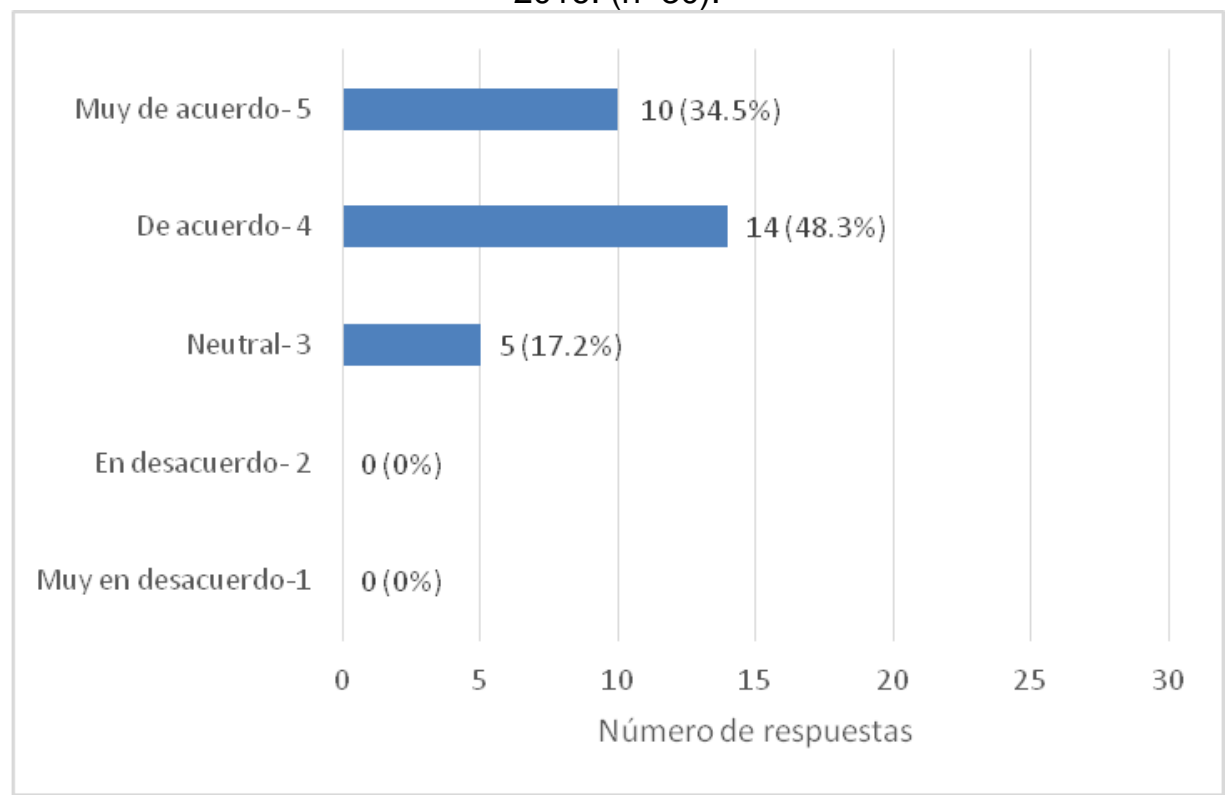

Fuente: Elaboración propia con la información recolectada, 2016. 
La preferencia hacia los métodos comunicativos de enseñanza se puede explicar por la percepción del estudiantado sobre los recursos que les sirvieron a ellos mismos para aprender una LE. La mayoría de sus docentes usó un método tradicional, lo que genera un rechazo retrospectivo que en ocasiones es muy explícito: "yo no haría lo que hicieron conmigo; que toda la clase de LE está basada en gramática" (grupo 3). En las entrevistas individuales se aportan algunas experiencias negativas concretas. Por ejemplo, la informante dos:

"Recuerdo una vez que tuve un trabajo que... era como escribir un cuento y teníamos que hablar de unos patos, de lo que pasaba con ellos, de lo que ellos hacían (...). Entiendo que hubiera sido mejor si lo hubiéramos aplicado a la vida real, ahora lo veo y pienso que era muy escuelero, por decirlo así, muy fuera de contexto".

De hecho, los encuestados perciben que aprendieron más en interacción con la lengua: las dos opciones más votadas reconocen la importancia de entrar en contacto con muestras de lengua reales $(M=4.58)$ y de utilizar la lengua en contextos auténticos $(M=4.75)$. Estos datos se confirman en las discusiones grupales, como la sostenida por el grupo dos:

14: "hay que llevar la gramática, ojalá, a un contexto de la vida real, para que el estudiante vea que tiene una aplicación en la vida real, eso lo empieza a motivar al estudiante (...)".

12: “(...) lo que nunca haría es remitir al estudiante a un libro de gramática, jamás haría eso, un libro de gramática pura, porque no le interesa, no es de su campo disciplinar, y mucho menos le interesa (...)".

11: "He tenido también varias experiencias con la aproximación a la gramática (...), lo que buscamos es que el estudiante se comunique, que se exprese en la lengua extranjera (...). Yo vine a aprender la lengua cuando tuve que comunicarme, cuando tuve que comunicar algo específico, ahí sí aprendí la estructura gramatical”.

Por otro lado, no se abandonan los recursos más ligados a una visión tradicional, ya que aspectos como el estudio individual reciben una puntuación relativamente alta $(\mathrm{M}=4.17)$, la memorización $(\mathrm{M}=3.58)$ y la realización de ejercicios en libros de texto o fotocopias $(\mathrm{M}=3.79)$.

Estos resultados se pueden relacionar de forma directa con los referidos a los recursos que elegirían los encuestados para enseñar gramática. Así, una gran mayoría apoya la idea 
de permitirle al estudiantado usar la lengua que están aprendiendo $(M=4.79)$, y es también alto el promedio otorgado a la idea de que el trabajo gramatical solo tiene sentido mediante actividades comunicativas $(M=4.0)$. En esta línea, las conclusiones de las discusiones gramaticales indican que la gramática no es en sí misma un problema, sino su enseñanza en contextos reales. Esto explica que el promedio obtenido en la pregunta sobre la utilidad de los ejercicios de drill sea relativamente bajo $(M=3.17)$. Este tipo de actividades son comparadas con "un robot llenando espacios en blanco", lo que no permite "comprender el uso real de la estructura" (grupo 3). En las gráficas 5 y 6 , se observa el diferente apoyo dado a las actividades comunicativas y a las de drill:

\section{Gráfica 5}

Distribución porcentual por nivel de acuerdo de los docentes en formación del Instituto Caro y Cuervo de Bogotá, ante la afirmación "El trabajo gramatical solo tiene sentido mediante actividades comunicativas", 2016. $(n=30)$.

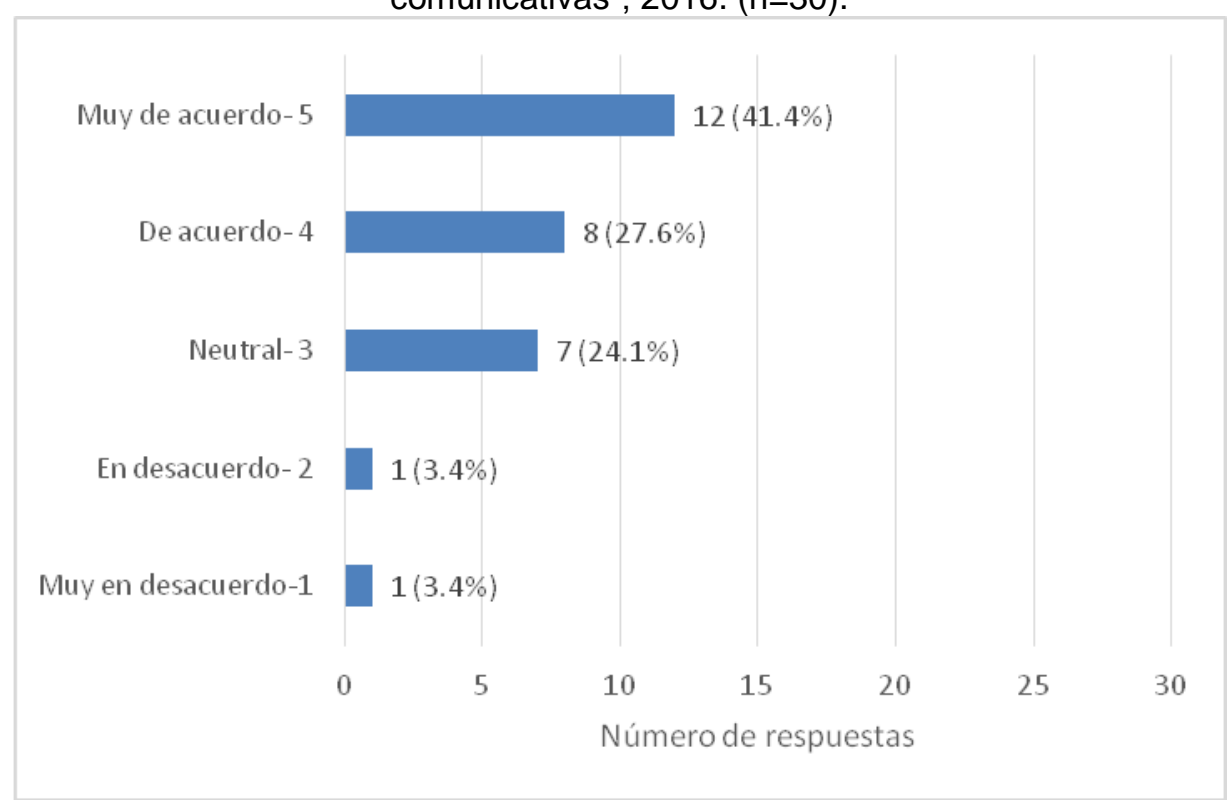

Fuente: Elaboración propia con la información recolectada, 2016. 


\section{Gráfica 6}

Distribución porcentual por nivel de acuerdo de los docentes en formación del Instituto Caro y Cuervo de Bogotá, ante la afirmación "Los ejercicios de drill (completar huecos) son una herramienta muy útil en la enseñanza de la gramática", 2016. ( $n=30)$.

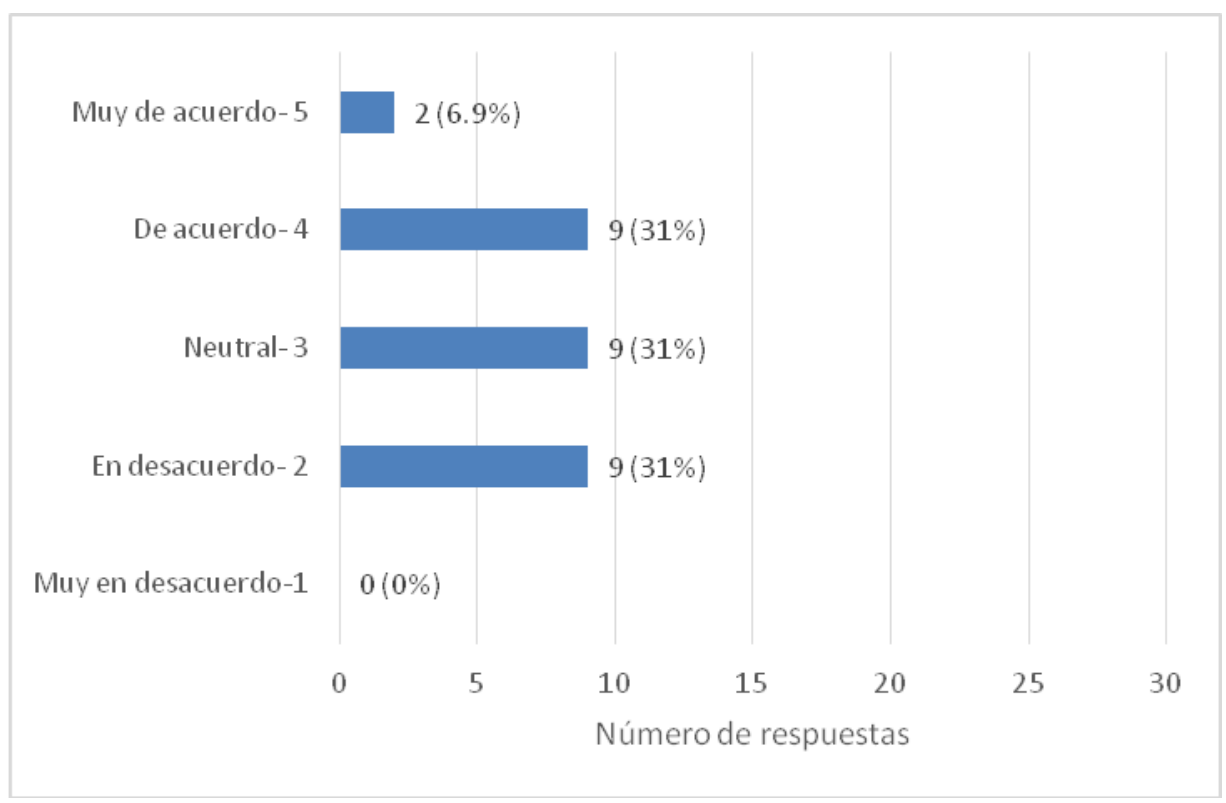

Fuente: Elaboración propia con la información recolectada, 2016.

El predominio de las opciones más comunicativas se confirma de nuevo en las preguntas sobre si el estudiantado debería prestar atención a la forma o al uso de la lengua. Aunque los promedios obtenidos son bastante altos, la atención al uso de la lengua es preferida por una mayoría ( $M=4.79$ frente a $M=4.31$ otorgado a la atención a la forma). En general, los entrevistados coinciden con la afirmación del grupo 5, en el sentido de que "es importante la manera en la que la gramática se presenta, debe enseñarse la funcionalidad, no solo una cantidad de reglas". La mayoría de las entrevistas individuales recogen esta misma idea, se destaca el deseo de que el estudiantado aprenda de forma contextualizada e infiriera de esta manera el funcionamiento de la gramática.

De forma análoga, existe una tendencia a destacar el papel activo del cuerpo estudiantil en el aprendizaje de la gramática. Se considera importante que la persona docente domine los contenidos gramaticales $(M=4.89)$, pero también que el estudiantado desempeñe un papel activo en la construcción del conocimiento $(M=4.68)$ y que pueda poner en práctica la lengua que está aprendiendo $(M=4.96)$. Los entrevistados lo explican de esta manera: "Las explicaciones gramaticales no son exclusivas del profesor, los estudiantes pueden indagar e investigar" (grupo 3), y añaden diferentes fuentes que facilitan el aprendizaje de la gramática más allá de la figura docente, como son los recursos virtuales. 
Además, se otorga más importancia a la reflexión gramatical del propio estudiantado $(\mathrm{M}=3.82)$ que a la elaboración de una secuencia didáctica clásica $(M=3.17)$. En este contexto, el papel de la persona docente sería, sobre todo, el de retroalimentar al estudiantado ( $M=4.86)$ y, en menor medida, corregirles $(M=4.24)$. En los debates grupales se reconoce que quien enseña es un guía que "resuelve dudas más que presenta" (grupo 2), aunque no falta la visión contraria: "Me gusta que tengan los conceptos claros y así es como enseño" (grupo 4). En las entrevistas individuales, de hecho, se percibe una inquietud por no conocer en profundidad la gramática española, como por ejemplo:

"Personalmente creo que el problema grande ahí es que como hablante nativo del español de pronto no es tan consciente de las estructuras que uno usa... la falta de conocimiento sobre esas estructuras a mi parecer es el problema más grande" (Informante 6).

Otros datos de la encuesta ponen de manifiesto el deseo de cambio respecto a planteamientos tradicionales de enseñanza de la gramática. De esta manera, se cuestiona el papel central del libro de texto (que solo obtiene $M=3.06$ en la pregunta sobre su importancia) y, en cambio, se resalta la importancia del clima afectivo para el aprendizaje $(\mathrm{M}=4.10)$. De hecho, se percibe la aspiración de acabar con la idea de la enseñanza de la gramática como una obligación pesada: "si se conecta con propósitos comunicativos reales deja de ser aburrida y comienza a ser interesante, ese es el objetivo del estudiante, de la institución" (grupo 2). En general, se reconoce la importancia de la motivación del estudiantado y de su papel activo en el aprendizaje. El papel activo estudiantil y el cambio de enfoque se ve claramente en afirmaciones como:

"Todo depende de los objetivos del estudiante, porque si no le interesa la gramática al estudiante no es necesario. Son necesarias [las explicaciones] si el estudiante lo ve asi" (grupo 1),

"Hay que tener en cuenta la edad de los estudiantes, a los adultos les gusta mucho la gramática, las explicaciones claras de uso, pero a los niños y jóvenes les gusta más deducir y los nombres y los conceptos son muy complicados para ellos, ellos quieren saber cómo usarlo mas no los conceptos" (grupo 2).

En esta misma línea acerca de prestarle atención a la realidad del estudiantado, se puede contemplar el deseo de prestar atención a las particularidades de la LM del 
estudiantado en las explicaciones gramaticales del español (solo el 16\% las evitaría en la clase de ELE), así como el rechazo al uso del metalenguaje (con un apoyo de solamente $M=2.96)$. Los encuestados inciden en este aspecto con frases como "el estudiante no debe conocer el metalenguaje sino usar la lengua" (grupo 5), y concluyen a partir de su experiencia que "hay que explicar la gramática con ejemplos reales y cercanos a los estudiantes y no con metalenguaje" (grupo 1), lo que no está exento de dificultades: "yo aún no he encontrado como explicar la gramática sin esos conceptos" (grupo 1).

\section{Conclusiones}

Los resultados de esta investigación muestran una clara disposición del estudiantado investigado a contemplar la orientación comunicativa en la enseñanza del español como lengua extranjera. Este hecho resulta especialmente significativo, pues se da en estudiantes que aún no han comenzado su formación especializada en ELE. Entendemos que esta actitud hacia un tratamiento comunicativo de la gramática se puede explicar desde la propia experiencia como aprendices de idiomas. Pese a que la mayor parte del estudiantado dice haber tenido un aprendizaje de lenguas tradicional, se destacan aspectos relacionados con el uso real de la lengua, tanto en los medios utilizados (canciones, cine, etc.) como en la finalidad de dicho uso.

La sensibilidad hacia los aspectos comunicativos no evita que se posea una visión "tradicional" acerca de la gramática y su papel en el proceso de enseñanza y aprendizaje. La manera restrictiva en que se entiende el papel de la gramática, relacionada con la norma y la corrección, podría deberse al contacto con las gramáticas descriptivas. Será una labor de los programas de formación en ELE aportar diferentes dimensiones de la gramática, con atención a sus contenidos comunicativos, cognitivos y pedagógicos, y proporcionar herramientas didácticas para poner en práctica este cambio de orientación gramatical.

Por último, un aspecto muy destacado de esta investigación es la preocupación que las personas informantes muestran hacia el estudiantado en sí, hacia sus necesidades e intereses. Este hecho repercutirá, sin duda, de manera positiva tanto en el proceso de formación profesional en ELE como en la futura práctica docente.

Los resultados de esta investigación, limitada por el número de los informantes, podrían contrastarse con otros que estudiasen creencias sobre gramática en diferentes cursos de formación, instituciones o regiones. De esta manera, se lograría un conjunto de datos más significativo $\mathrm{y}$, tal vez, se obtendrían matices diferenciadores dependientes de 
diversos contextos. Estamos, por lo tanto, ante la posibilidad de confirmar si se están generando, y dónde, nuevas tendencias en la concepción de la enseñanza de gramática, más orientada hacia los elementos comunicativos y las necesidades estudiantiles; o si se trata, solamente, de un hallazgo puntual localizado en la muestra estudiada.

\section{Referencias}

Andrews, Stephen. (1999). All these like little name things': a comparative study of language teachers' explicit knowledge of grammar and grammatical terminology. Language Awareness, 8(3/4), 143-59.

Barcelos, Ana María y Kalaja, Paula. (2011). Introduction to Beliefs about SLA revisited. System, 39(3), 281-289.

Berry, Roger. (1997). Teachers' awareness of learners' knowledge: The case of metalinguistic terminology. Language Awareness, 6(2-3), 136-146.

Berry, Roger. (2014). Investigating Language Awareness: The Role of Terminology. En Andrzej Łyda y Konrad Szczesniak (Eds.), Awareness in Action, Second Language Learning and Teaching (pp. 21-33). Cham, Suiza: Springer International Publishing.

Borg, Simon. (1999). Teachers theories in grammar teaching. ELT Journal, 53(3), 157-167.

Borg, Simon. (2001). Self perception and practice in teaching grammar. ELT Journal, 55(1), 21-29.

Borg, Simon. (2003). Teacher cognition in language teaching: A review of research on what laguage teachers think, know, believe, and do. Language teaching, 36(02), 81-109.

Borg, Simon. (2005). Experience, knowledge about language and classroom practice in teaching grammar. En Nat Bartels (Ed.), Applied Linguistics and Language Teacher Education (pp. 325-340). New York: Springer.

Borg, Simon. (2006). Teacher Cognition and Language Education: Research and Practice. Londres: Continuum.

Burgess, John y Etherington, Siân. (2002). Focus on grammatical form: explicit or implicit. System, 30(4), 433-458.

Chandler, Paul, Robinson, Peter y Peter, Noyes. (1988). The level of linguistic knowledge and awareness amongst students training to be primary teachers. Language and Education 2(3), 161-173.

Clarke, David y Hollingsworth, Hilary. (2002). Elaborating a model of teacher professional growth. Teaching and Teacher Education, 18(8), 947-967

De Witt, Paul, Birrell, James, Egan, Winston, Cook, Paul, Ostlund, Margaret y Young, James. (1998). Professional development schools and teacher educators' beliefs: Challenges and change. Teacher Education Quarterly, 25(2), 63-80. 
Eisenstein-Ebsworth, Miriam, y Schweers, William. (1997). What Researchers Say and Practitioners Do. Perspectives on Conscious Grammar Instruction in the ESL Classroom. Applied language learning, 8(2), 237-260.

Farrell, Thomas y Lim Poh Choo, Patricia. (2005). Conceptions of Grammar Teaching: A Case Study of Teachers' Beliefs and Classroom Practices. TESL-EJ, 9(2), 1-13.

Gabillon, Zehra. (noviembre, 2013). A Synopsis of L2 Teacher Belief Research. En Tomorrow People Organization (Organizadores). Belgrade International Conference on Education 2013, Belgrade, Serbia. Recuperado de https://hal.archivesouvertes.fr/file/index/docid/940593/filename/1. Z.Gabillon A Synopsis of L2 Teacher Belief Research BICE Conference.pdf

Johnston, Bill y Goettsch, Karin. (2000). Insearch of the knowledge base of language teaching: Explanations by experienced teachers. Canadian Modern Language Review, 56(4).

Kalaja, Paula y Barcelos, Ana María. (2003). Beliefs about SLA: New Research Approaches. Dordrecht: Kluwer Academic Press.

Nazari, Ahmad y Allahyar, Negah. (2012). Grammar Teaching Revisited: EFL Teachers between Grammar Abstinence and Formal Grammar Teaching. Australian Journal of Teacher Education, 37(2), 73-87.

Pahissa, Isabel y Tragant, Elsa. (2009). Grammar and the non-native secondary school teacher in Catalonia. Language Awareness, 18(1), 47-60.

Ramos Méndez, Carmen. (2007). El pensamiento de los aprendientes en torno a cómo se aprende una lengua: dimensiones individuales y culturales. España: Ministerio de Educación.

Santiago Sánchez, Hugo. (2010). An investigation into the relationships among experience, teacher cognition, context, and classroom practice in EFL grammar teaching in Argentina (Tesis para optar por el grado de Doctorado). The University of Warwick, Coventry Reino Unido.

Schulz, Renate. (2001). Cultural differences in student and teacher perceptions concerning the role of grammar instruction and corrective feedback: USA-Colombia. The Modern Language Journal, 85(2), 244-258.

Uysal, Hacer y Bardakci, Mehmet. (2014). Teacher beliefs and practices of grammar teaching: focusing on meaning, form, or forms? South African Journal of Education, 34(1), 1-16.

Williamson, John y Hardman, Frank. (1995). Time for refilling the bath? A study of primary student-teachers' grammatical knowledge. Language and Education, 9(2), 117-34.

Wu, Kam Yin. (2006). Teacher beliefs and grammar teaching practices: Case studies of four ESL teachers. (Tesis para optar por el grado de Doctorado). Hong Kong University. 